\title{
Invasive Hydatidiform Mole
}

National Cancer Institute

\section{Source}

National Cancer Institute. Invasive Hydatidiform Mole. NCI Thesaurus. Code C6985.

A complete hydatidiform mole or very rarely a partial mole that invades the myometrium. 